\title{
Elegancy: An open-source and intuitive cloud-based electronic
}

\section{laboratory notebook}

\author{
Chih-Wei Huang ${ }^{\mathrm{a}}$, Wei-Hsuan Chuang ${ }^{\mathrm{a}}$, Shu-Hwa Chen ${ }^{\mathrm{b}}$, Chung-Yen Lin ${ }^{\mathrm{a}}$ \\ ${ }^{a}$ Institute of Information Science, Academia Sinica, Taipei, TAIWAN \\ ${ }^{\mathrm{b}}$ TMU Research Center of Cancer Translational Medicine, Taipei Medical University, Taipei, TAIWAN
}

\begin{abstract}
We introduce an electronic laboratory notebook, Elegancy, a cloud-based and standalone web service distributed as a Docker image. Elegancy allows researchers to digitize and safeguard their documentations in laboratories. It helps the scholarly community in gathering evidence, sharing information, reorganizing knowledge, and creating laboratory works with greater ease and security.
\end{abstract}

\section{Introduction}

In the scientific community, paper-based laboratory notebooks have long been the dominant platform for recording experimental data. However, data written on paper cannot easily be searched, shared, or backed up, and it can easily be misplaced or lost. As stated in one study, up to $17 \%$ of undigitized research data are lost per year ${ }^{1}$. In addition, with the increase of automated and modernized lab equipment, massive volumes of data are generated in a digital rather than physical format. These issues have given rise to Electronic Laboratory Notebooks (ELN), single platforms capable of storing, organizing, searching, and sharing experimental data. ELNs significantly reduce human error in data archiving, and they make sharing information more convenient. Users can gain access to an ELN anytime and anywhere. Because ELNs can be saved locally or in the cloud, data is backed up multiple times. A plethora of ELNs are in the market, each with its own merits ${ }^{2}$. For example, SciNote (https://www.scinote.net/) is fairly intuitive and can be installed locally or through Docker containers. Similarly, eLabFTW ${ }^{3}$ is an open-source software and supports both local and cloud hosting. eLabFTW also offers a Docker image. However, as with most commercial ELNs, they provide limited storage and only a few functions in their unpaid version.

Thus, we created a cloud-based ELN, Elegancy, which employs a hybrid system of Drupal and our developed modules. Users can install it either on local machines or on servers, and they can access it using a web browser on any mobile device. It is open-source and easy to install and navigate with all functions available in the same package. Most importantly, Elegancy is entirely free. Elegancy is suitable for laboratories of any type and has no limit on the number of users. Elegancy would, therefore, be of great use in information sharing and lab work management in the research community.

\section{Implementation}

Elegancy is developed based on Drupal 7 (https://www.drupal.org/drupal-7.0), which is an open-source content management platform. In total, 190 operating modules are in the system, including core components; the management interface; and those providing content creation, data sharing, search engine capacity, panel construction, multilingual support, information safeguarding, and backup and restore capabilities. Among those modules, 170 are modified from the built-in modules and 20 are constructed by our research and development team. MySQL 8.0.25 (https://www.mysql.com/ ) was used to establish the database, and Apache HTTP Server 2.4.41 (https://httpd.apache.org/) was used to set up the web server. The programming languages implemented here are PHP 7.1.33 (https://www.php.net), JAVA runtime environment (JRE) 8.0.1. (https://www.oracle.com/java/technologies/javasejre8-downloads.html), HTML and CSS.

To ensure that even users without a background in computer science can easily navigate Elegancy, our team focused on developing an ELN that can be swiftly installed by following simple instructions. Elegancy supports both local and cloud installation. With Inno Setup as the foundation for local installation, we wrapped software packages, such as Apache, MySQL, and PHP, into our installation program. Because Java was used in writing programs related to ELN management, which are required for initiating and shutting down ELNs, we also included JRE in the package. Local download is currently available for Windows only. However, with the Docker image that we provide, the aforementioned necessary software packages are wrapped with the Docker developer, allowing users to install and build their ELNs on different operating systems (e.g., Linux and Windows), DOCKER environments, NAS drives, or cloud computing platforms. To save users from having to learn complicated Docker commands, we offer a Shell Script with the required commands for installation written inside.

\section{Results}

Based on a recent review of the highest ranked ELNs ${ }^{4}$ and a comparison table between several ELNs ${ }^{5}$, we constructed a matrix (see Supplementary Table 1) summarizing the advantages of Elegancy and described them as follows. Elaboration on Elegancy's functions is provided in Figure 1 and in Supplementary Note 1.

1. User experience: 
Elegancy is easy to set up. Users can build their ELN by following the simple instructions and tutorial videos provided on our website. The graphical user interface is intuitive and uncluttered. Options are displayed on the top of the panel, including a calendar that also serves as a timestamp. Users can also track the consumption of either office supplies or chemical solvents. English is the default language of Elegancy, with Mandarin and Japanese as built-in options. Users who have the resources for translation may add other languages. We provide multiple background themes for users to choose from. With regard to post-editing, the text editor is similar to those in commonly used programs and applications, such as Microsoft Word or Google Documents. We included MathType and ChemType plugins from CKEditor to allow for the creation of mathematical equations and chemical formulas. A module for drawing chemical structures is also provided. Users can insert code, images, or tables and attach links to uploaded files, multimedia, or other posts. All forum posts can also be exported as PDFs, and their source code is open for template modification if necessary. Elegancy has further been developed to facilitate collaboration. Users can share posts and meeting invitations with other users through email from our ELN. Elegancy also includes a module that analyzes the interactivity between members, which can be presented in charts.

\section{Interoperability and Flexibility:}

Elegancy is built based on Drupal, which allows users to add their personalized modules. It supports different operating systems, such as Windows, Mac OS, and Linux, and several web browsers, including Google Chrome, Firefox, Safari, IE, and Opera. We highly recommend choosing Elegancy's Docker image over local installation for several reasons: First, cloud backups can spare users the trouble of transferring or restoring databases between hardware storage devices. Second, it enhances data preservation security. Third, users can access their ELN from any device anytime and anywhere. Recently, we developed an optimized mobile device interface, and users can now upload photos, drawings, or recordings to their ELN directly from their phones. Although Elegancy is fit for all types of laboratories, these features will likely be highly beneficial to wet labs.

\section{Security and Pricing:}

Several aspects of the platform guarantee data security. For example, user access is divided into different levels of authorities: Guest, Lab Member, Project Leader, and Site Manager (see Supplementary Note 1 Section 3.1). The use of the Docker images also ensures remote data backup and easy database restoration. We also complied with the RFC 3161 Time-Stamp Protocol ${ }^{6}$ and further applied audit trials for documentation control following two of the three main factors regulated by CFR Title 21 Part 11 ${ }^{7}$ : Data Security and Electronic Records. All events are recorded with the exact username, date, and time; and only site managers have the access to these records. In most ELNs, the free version usually includes limited functions and resources. However, with Elegancy, all the necessary functionalities are included, entirely free of charge, in the same package.

\section{(2) Multi-functional Modules in ELN (Elegancy)}

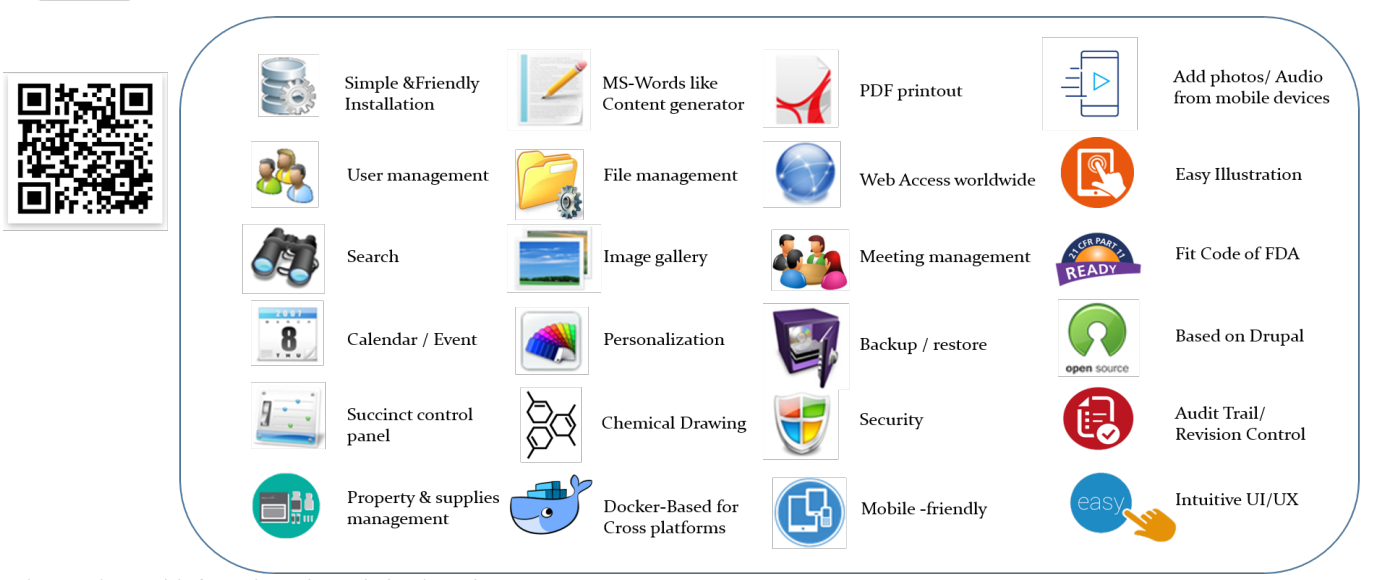

Figure 1. Multi-functional modules in Elegancy

\section{Conclusion}

Elegancy offers a multitude of functions for all types of research labs. It supports both local and cloud hosting, both of which are available with a Docker image. The installation steps are simple, and the user interface is intuitive. Tutorials of Elegancy's basic functions and an introduction video of the overall features are provided online. Elegancy is compatible with several operating system layers and can be accessed anywhere through any electronic device. It also includes special features specifically for mobile device use. Most importantly, Elegancy is charge-free for all users.

Elegancy continues to develop and is expected to receive several feature improvements: First, our team plans to add an auto-reminder function for timeconsuming programs or experiments. Second, modern laboratory equipment typically supports the exporting of results in a digital format. Therefore, we will attempt to develop a function that allows for the direct input of experiment data directly into the ELN. Third, Jupyter notebook (https://jupyter.org/) is currently widely used and indispensable to many laboratories. Thus, binding Jupyter notebook to our ELN would benefit those who are highly dependent on it. Finally, our team hopes to import the protocols from web pages, such as Protocols.io (https://www.protocols.io/), to our ELN to assist in extremely complex experiments with numerous detailed steps.

\section{Acknowledgements}

This manuscript was edited by Wallace Academic Editing.

\section{Data Availability}

Docker image of Elegancy is freely accessible at both https://hub.docker.com/r/lsbnb/eln and https://github.com/lsbnb/eln 


\section{References}

1. Vines T. H. et al. (2014). The Availability of Research Data Declines Rapidly with Article Age. Current Biology, 24, 94-97.

2. Huchet B. (2021). 2021 Review of the Best Electronic Laboratory Notebooks. Labs Explorer.

3. Hewera M, Hänggi D, Gerlach B and Kahlert UD. eLabFTW as an Open Science tool to improve the quality and translation of preclinical research [version 3; peer review: 2 approved]. F1000Research 2021, 10:292 (https://doi.org/10.12688/f1000research.52157.3 )

4. Kovac K. (2021). Best Electronic Lab Notebook (ELN) Review For Your Lab in 2021. Splice.

5. Harvard Longwood Medical Area Research Data Management Working Group. (2021). Electronic Lab Notebook Comparison Matrix. Zenodo. https://doi.org/10.5281/zenodo.4723753

6. Adams C. et al. (2001) Internet X.509 Public Key Infrastructure Time-Stamp Protocol (TSP).

7. U.S. Food and Drug Administration (2020). Code of Federal Regulations Title 21 Part 11. 